\title{
CD13 Induces Autophagy to Promote Hepatocellular Carcinoma Cell Chemoresistance Through the P38/Hsp27/CREB/ ATG7 Pathway ${ }^{\circledR}$
}

\author{
Yan Zhao, ${ }^{1}$ Huina Wu, ${ }^{1}$ Xiaoyan Xing, Yuqian Ma, Shengping Ji, Xinyue Xu, Xin Zhao, \\ Sensen Wang, Wenyan Jiang, Chunyan Fang, Lei Zhang, Fang Yan, and Xuejian Wang \\ School of Pharmacy, Weifang Medical University, Weifang, Shandong, China (Y.Z., H.W., X.Xi., Y.M., S.J., X.Xu., X.Z., S.W., W.J., \\ C.F., L.Z., F.Y., X.W.) and Department of pharmacy, Southwestern Lu Hospital, Liaocheng, Shandong, China (H.W.)
}

Received February 12, 2020; accepted June 17, 2020

\begin{abstract}
The chemoresistance of hepatocellular carcinoma $(\mathrm{HCC})$ is a serious problem that directly hinders the effect of chemotherapeutic agents. We previously reported that Aminopeptidase N (CD13) inhibition can enhance the cytotoxic efficacy of chemotherapy agents. In the present study, we use liver cancer cells to explore the molecular mechanism accounting for the relationship between CD13 and chemoresistance. We demonstrate that CD13 overexpression activates the P38/heat shock protein 27/ CAMP response element-binding protein (CREB) signaling pathway to limit the efficacy of cytotoxic agents. Moreover, blockade of P38 or CREB sensitizes HCC cells to 5-fluorouracil. Then we reveal that CREB binds to the autophagy related 7 (ATG7) promoter to induce autophagy and promote HCC cell chemoresistance. CD13 inhibition also downregulates the expression
\end{abstract}

of ATG7, autophagy, and tumor cell growth in vivo. Overall, the combination a CD13 inhibitor and chemotherapeutic agents may be a potential strategy for overcoming drug resistance in HCC.

\section{SIGNIFICANCE STATEMENT}

Our study demonstrates that Aminopeptidase N (CD13) promotes hepatocellular carcinoma $(\mathrm{HCC})$ cell chemoresistance via the $\mathrm{P} 38 /$ heat shock protein $27 / \mathrm{cAMP}$ response element-binding protein (CREB) pathway. CREB regulates autophagy related 7 transcription and expression to induce autophagy. Our results collectively suggest that CD13 may serve as a potential target for overcoming HCC resistance.

\section{Introduction}

Hepatocellular carcinoma (HCC), also known as malignant hepatoma, is characterized by high morbidity and mortality rates, and it is one of the most common malignancies worldwide (Xu et al., 2012; Guo et al., 2017). Recent developments in treatment methods for HCC, including surgical resection and chemotherapy, have shown considerable efficacy (Wei et al., 2014; Zhang et al., 2015). Surgical resection is mainly applicable to early-stage patients, whereas chemotherapy is a crucial strategy for advanced patients (Raza and Sood, 2014). However, the rates of intrahepatic recurrence and

This work was supported by the National Natural Science Foundation of China [Grant 81503108]; Qing Chuang science and technology plan of colleges and universities in Shandong Province [Grant 2019KJM001]; Project of Shandong Province Higher Educational Science and Technology Program [Grant J17KA255]; and University Students Science and Technology Innovation Fund Project [Grants 201910438027X and KX2019025].

The authors declare that they have no conflict of interest, financial or otherwise, in this study.

${ }^{1}$ Y.Z. and H.W. contributed equally to this work.

https://doi.org/10.1124/jpet.120.265637.

S This article has supplemental material available at jpet.aspetjournals.org mortality remain high because of the occurrence of resistance to chemotherapeutic drugs (Yamashita et al., 2016). Therefore, examining the mechanism of drug resistance and exploring new therapeutic targets that could reverse the drug resistance of HCC cells may provide novel strategies to treat the disease.

Aminopeptidase N (CD13) (EC 3.4.11.2) is a type II zincbinding transmembrane metallopeptidase with the structure of a noncovalent bound homodimer found on the exterior of cellular membranes (Mina-Osorio, 2008). CD13 is highly expressed in various types of tumors, including renal carcinoma, lung cancer, colon cancer, and prostate cancer (Ishii et al., 2001; Hashida et al., 2002; Sun et al., 2015). CD13 plays a vital role in tumor angiogenesis, invasion, and metastasis (Sato, 2003). A recent study demonstrates that the enzyme acts as a marker of semiquiescent cancer stem cells (CSCs) in human liver cancer (Haraguchi et al., 2010). In an in vivo transplantation model of mice, the volume of solid tumors significantly decreased after combination treatment with 5fluorouracil (5FU) and ubenimex, a CD13 inhibitor (Nagano et al., 2012). We previously reported that ubenimex and

ABBREVIATIONS: 3-MA, 3-methyladenine; ATG7, autophagy related 7; CD13, aminopeptidase N MTT methyl thiazolyl tetrazolium; ChIP, chromatin immunoprecipitation; CSC, semiquiescent cancer stem cells; CREB, cAMP response element-binding protein; 5FU, 5-fluorouracil; HCC, heptaocellular carcinoma; Hsp27, heat shock protein 27; MAPK, mitogen-activated protein kinase; OD, optional density; p, phosphorylated; ROS, reactive oxygen species; qPCR, quantitative polymerase chain reaction; qRT-PCR, quantitative reverse-transcription polymerase chain reaction. 
a neutralizing antibody could enhance the antitumor effects of $5 \mathrm{FU}$ and other cytotoxic agents on HCC cell lines by upregulating the level of intracellular reactive oxygen species (ROS). Whereas CD13 overexpression could strengthen the resistance of PLC/PRF/5 cells to chemotherapeutic drugs, knockdown of CD13 remarkably increases the sensitivity of PLC/ PRF/5 cells to antitumor drugs (Zhang et al., 2018).

Autophagy is an evolutionarily conserved "self-degradative" process by which cells clear long-lived or misfolded proteins, protein aggregates, and damaged organelles to maintain cellular homeostasis under low-oxygen, nutrient-deprived, and stressed conditions (Chen et al., 2011; Levy et al., 2017; Hu et al., 2019). Some studies show that autophagy functions as a tumor suppressor in the early stages of tumor initiation. Autophagy has also been noted to facilitate cancer cell survival at advanced stages of tumor development, which indicates that autophagy plays a dual role in cancer cells (Huang et al., 2018). Data also demonstrate that autophagy induction promotes chemoresistance to facilitate cell survival, which suggests that suppression of autophagy may enhance the sensitivity of cancer cells to chemotherapeutic drugs (O'Donovan et al., 2011; Pan et al., 2014).

Accumulating evidence indicates that the contribution of CD13 is closely related to the resistance of HCC cells to chemotherapeutic drugs, but the relevant molecular mechanism remains unknown. In the present study, we demonstrate that CD13 activates P38 protein kinase (P38)/heat shock protein 27 (Hsp27)/cAMP responsive element binding protein (CREB)-mediated autophagy related 7 (ATG7) transcription and expression to induce autophagy and promote the chemoresistance of HCC cells.

\section{Materials and Methods}

Cell Culture. The human hepatoma cell lines PLC/PRF/5 and Huh7 were obtained from the Cell Bank of Shanghai (Shanghai, China). We constructed stable CD13 overexpression (PLC/PRF/5/ CD13) and knockdown (PLC/PRF/5/CD13 short hairpin RNA) PLC/ $\mathrm{PRF} / 5$ cells by using the lentiviral vector (Genechem, China) and puromycin screening (Zhang et al., 2018). The obtained PLC/PRF/5 and Huh7 cell lines were respectively maintained in modified Eagle's medium and Dulbecco's modified Eagle's medium supplemented with $10 \%$ FBS and penicillin-streptomycin in a humidified incubator under of $5 \% \mathrm{CO}_{2}$ at $37^{\circ} \mathrm{C}$.

Western Blot Analysis. Cells were washed with $1 \times$ PBS and lysed in RIPA buffer containing protease inhibitor cocktail (SigmaAldrich). Protein concentrations were determined by using Bradford protein quantification and were subjected to SDS-PAGE. The gel was cut according to the relevant markers, and target proteins were transferred onto polyvinylidene difluoride membranes (Millipore). The target proteins were determined by Western blot with their respective specific antibodies. $\beta$-actin or total protein was used as an internal control. Blots were visualized by an enhanced chemiluminescence kit (Millipore). Densitometry for Western blot was performed by using AlphaEaseFC-v4.0.0 software. The following antibodies were used in this study: CD13 (dilution of 1:1200, catalog number sc-51522; Santa Cruz Biotechnology, China), P38 (dilution of 1:1000, catalog number sc-271120; Santa Cruz Biotechnology), p-P38 (dilution of 1: 1000, catalog number sc-166182; Santa Cruz Biotechnology), Hsp27 (dilution of 1:1000, catalog number sc-13132; Santa Cruz Biotechnology), p-Hsp27 (dilution of 1:1000, catalog number sc-101700; Santa Cruz Biotechnology), CREB (dilution of 1:1000, catalog number sc-240; Santa Cruz Biotechnology), p-CREB (dilution of 1:1000, catalog number sc-7978; Santa Cruz Biotechnology), ATG7 (dilution of 1:1000, catalog number 8558; Cell Signaling Technology), LC3 (dilution of 1:1000, catalog number
3868; Cell Signaling Technology), and $\beta$-actin (dilution of 1:1000, catalog number sc-1616; Santa Cruz Biotechnology).

MTT Assay. Cell viability was evaluated by Methyl Thiazolyl tetrazolium (MTT) assay. The PLC/PRF/5, Huh7, and PLC/PRF/5/ $5 F U$ cells were seeded in $100 \mu$ l of growth medium at a density of $5 \times$ $10^{\wedge}$ cells per well in 96 -well plates. After an overnight incubation, the cells were treated with specific reagents. After 48 hours of treatment, the cells were added with MTT solution (Beijing Solarbio Science and Technology, China) and incubated for another 4 hours at $37^{\circ} \mathrm{C}$. The supernatant was discarded, and $100 \mu \mathrm{l}$ of dimethyl sulfoxide was added to each well. The optional density (OD) of the solutions in the wells was measured at 570 and $630 \mathrm{~nm}$ by using a multifunctional microplate reader (SpectraMax M5; Molecular Devices, Sunnyvale, $\mathrm{CA}$ ). The inhibition rate was calculated via the formula (OD control OD tested)/OD control $\times 100 \%$, where the OD is the mean value of three replicate wells. The $\mathrm{IC}_{50}$ values were determined by using ORIGIN 8 software (OriginLab Corporation, Northampton, MA).

Quantitative Real-Time Polymerase Chain Reaction. Total RNA was extracted with Trizol reagent (Ambion), and the RNA concentration was detected by a Nano Drop instrument. Singlestrand cDNA was reverse-transcribed by using a HiFiscript cDNA Synthesis Kit (CWBIO, China) according to the manufacturer's protocol. The cDNA was then amplified with a SYBR Green mixture (CWBIO) and gene-specific primers. The primers used for quantitative reverse-transcription polymerase chain reaction (qRT-PCR) were as follows: CD13 forward, 5'-GGGCAGGGAAGAGGGTGGAC-3'; reverse, 5'-AGGAAGGTGGCTGAGAGTGGATG-3'; ATG7 forward, 5' CTGCCAGCTCGCTTAACATTG-3'; reverse, 5'-CTTGTTGAGGAG TACAGGGTTTT-3'. Finally, -qRT-PCR- was performed on an Authorized Thermal Cycler (LighterCycler480 Systems), and the relative mRNA level of the target gene was measured via the $2^{-\Delta \Delta \mathrm{CT}}$ method. Glyceraldehyde-3-phosphate dehydrogenase (GAPDH) was used as an internal control, and each reaction was performed in triplicate.

Chromatin Immunoprecipitation. Chromatin immunoprecipitation (ChIP) assay was performed by using an EZ-Magna CHIPTMA/ G Kit (17-10086; Millipore) according to the manufacturer's standard protocol. Briefly, $1 \times 10^{\wedge}$ of PLC/PRF/5 and PLC/PRF/5/5FU cells were fixed in $1 \%$ formaldehyde and incubated for 10 minutes at $37^{\circ} \mathrm{C}$. Then unreacted formaldehyde was quenched by $10 \times$ glycine for 5 minutes at room temperature, and cells were collected with cold $1 \times$ PBS containing $1 \times$ protease inhibitor cocktail II. Cell and nuclear lysis buffers containing protease inhibitor cocktail II were added to each microfuge tube to isolate nuclei. Then the cells were sonicated to create chromatin fragments of 200-500 bp. Aliquots of $50 \mu \mathrm{l}$ were immunoprecipitated after supplementation with $1 \mu \mathrm{g}$ of anti-CREB (Cell Signaling Technology) or $1 \mu \mathrm{g}$ of normal mouse IgG and $20 \mu \mathrm{l}$ of fully resuspended protein $\mathrm{A} / \mathrm{G}$ magnetic beads. The mixture was placed on a rotator at $4^{\circ} \mathrm{C}$ and incubated overnight. Each tube was added to elution buffer and incubated at $62^{\circ} \mathrm{C}$ for 2 hours with shaking and $95^{\circ} \mathrm{C}$ for 10 minutes to reverse the protein/DNA complexes. IgG was used as a negative control. Isolated RNA was assayed by $1.5 \%$ agarose gel electrophresis and quantitative polymerase chain reaction (q-PCR). The ATG7 promoter region $(-2074$ to +100$)$ was divided into seven parts (Table 1$)$.

stubRFP-sensGFP-LC3B System. The PLC/PRF/5 cells were infected with the stubRFP-sensGFP-LC3B lentivirus (catalog number GRL2001; Genechem) and screened by puromycin. The fluorescence of the construct depended on the difference in $\mathrm{pH}$ between the acidic autolysosome and the neutral autophagosome. The progression of autophagic flux was assessd by monitoring the red/green (yellow) or red fluorescence of the cells. The infected cells were treated with 5FU/217505 for 24 hours, and their fluorescence was detected by laser scanning confocal microscopy.

Nude Mice Xenograft Model Assay. Four-week-old female nude mice were purchased from Hunan SJA Laboratory Animal Co., Ltd. (Hunan, China) and fed under specific pathogen-free conditions. PLC/ $\mathrm{PRF} / 5$ cells were collected and resuspended at a density of $5 \times 10^{\wedge} 7 / \mathrm{ml}$ with PBS. Then $100 \mu \mathrm{l}$ of the cell suspension was subcutaneously injected into the mice. After tumor implantation, the mice were randomly divided into six groups $(n=6)$ and administered PBS or 
TABLE 1

Primer of ATG7 promoter sets used in this study

\begin{tabular}{lcc}
\hline Region & Forward $\left(5^{\prime}-3^{\prime}\right)$ & Reverse $\left(5^{\prime}-3^{\prime}\right)$ \\
\hline-2074 to 1676 & ATGACTCCTGGTTGCCTCCCTTG & TGCTCACACAAAGCCTGTTTGGTAG \\
-1809 to 1412 & GTCAGCAAAGGGTGGTGGGATTATC & AGCAACTGAAGATCCGCAGAAGTG \\
-1502 to 1105 & GATCTTCAGTTGCTGCAAGCCATC & TCCGCAGATGTTTCAATGGTTAGGG \\
-1227 to 830 & CCTTCTCTCCCACCTCTCTCACTTC & AGGCGGGCGGATCACGAG \\
-901 to 504 & GCCTCCCAAAGTGCTGTGATTAC & GCCTCCCAAAGTGCTGTGATTAC \\
-599 to 202 & GCAGTCATCGCTCTTGTTGTTATG & GGGTGGCAGGTGTGGAGAG \\
-319 to +78 & GGGTGGCAGGTGTGGAGAG & TGGGAGGAACTTGAGTCGTGAGG \\
\hline
\end{tabular}

$5 \mathrm{FU}(25 \mathrm{mg} / \mathrm{kg}$ per day) and ubenimex $(74 \mathrm{mg} / \mathrm{kg}$ per day). Tumor volumes and body weights were monitored every 3 days. After 2 weeks, all mice were sacrificed and dissected, and tumor tissues were weighed. All animal experiments were approved by the guidelines of the Animal Care and Use Committee of Weifang Medical University.

Statistical Analysis. Data are presented as means \pm S.D. and were analyzed by using GraphPad Prism 6.0 statistical software. Student's $t$ test was used to analyze differences in the data between two groups. Multiple groups of data were compared by one-way ANOVA followed by Dunnett's test or two-way ANOVA followed by Bonferroni's test. $P \leq 0.05$ was considered to indicate statistical significance.

\section{Results}

CD13 Overexpression Results in HCC Cell Chemoresistance. To investigate the role of $\mathrm{CD} 13$ in cell resistance to cytotoxic agents, we first constructed the resistant cell line $\mathrm{PLC} / \mathrm{PRF} / 5 / 5 \mathrm{FU}$ by exposing PLC/PRF/5 cells to increasing gradient concentrations of $5 \mathrm{FU}$ over 4 months. MTT assay showed that the $\mathrm{IC}_{50}$ of the $\mathrm{PLC} / \mathrm{PRF} / 5 / 5 \mathrm{FU}$ cells was significantly higher than that of parental cells, thus suggesting that the desired resistant HCC cells had been successfully established (Fig. 1A). Western blot and qPCR were then employed to detect the expression of CD13 in $\mathrm{PLC} / \mathrm{PRF} / 5$ and the PLC/PRF/5/5FU cells. As shown in Fig. 1, B and C, CD13 expression was upregulated in the resistant cells. Small interfering RNA (SiRNA) targeting CD13 was employed to confirm whether the CD13 antibody would bind with CD13 antigen (Supplemental Fig. 1, A and B). In our previous study, CD13 overexpression or knockdown in PLC/PRF/5 cells was achieved by lentivirus infection supplemented with puromycin screening. The data indicated that CD13 overexpression induces HCC cell resistance to 5FU, Gemcitabine (GEM), cisplatin (cis-DDP), and Paclitaxel (PTX), whereas CD13 knockdown sensitizes cells to cytotoxic agents. The CD13 inhibitor ubenimex enhances the cytotoxicity of chemotherapeutic agents to HCC cells (Zhang et al., 2018). Overall, these results demonstrate that CD13 overexpression may contribute to $\mathrm{HCC}$ cell resistance to chemotherapeutic drugs.

Inhibition of the P38/Hsp27/CREB Signaling Pathway Sensitizes HCC Cell to 5FU. The molecular mechanism through which CD13 induces HCC cell resistance remains unknown. Hence, protein ChIP assay was employed to analyze the signaling pathway protein phosphorylation in PLC/PRF/5 and PLC/PRF/5/5FU cells. As shown in Fig. 2A, the protein phosphorylation expression levels of P38, Hsp27, and CREB were upregulated in resistant cells. In particular, the phosphorylation level of p-Hsp27 obviously increased. The data indicate that P38, Hsp27, and CREB may participate in the resistance of $\mathrm{HCC}$ cells to $5 \mathrm{FU}$. We investigated whether $5 \mathrm{FU}$ could upregulate the phosphorylation of $\mathrm{P} 38, \mathrm{Hsp} 27$, and CREB in PLC/PRF/5 cells. As shown in Fig. 2B, 5FU could

activate this pathway in a time-dependent manner. To determine whether the P38/Hsp27/CREB pathway is activated in PLC/PRF/5/5FU-resistant cells, we measured the phosphorylation of these proteins and found elevated p-P38, p-Hsp27, and p-CREB expression levels in resistant cells compared with parental cells. These results suggest that the P38/Hsp27/CREB pathway is positively correlated with $5 \mathrm{FU}$-induced HCC cell resistance (Fig. 2C). We supposed that P38, Hsp27, and CREB activation are triggered by $5 \mathrm{FU}$ induction to allow $\mathrm{HCC}$ cells to develop resistance. We examined this possibility by employing the P38 inhibitor SB202190 and the CREB inhibitor 217505 to detect their combined effect with $5 \mathrm{FU}$ on HCC cells. MTT assay showed that inhibition of P38 by SB202190 decreases the cell viability in a dose-dependent manner after $5 \mathrm{FU}$ treatment of PLC/PRF/5, Huh7, and PLC/PRF/5/5FU cells. This result suggests that P38 inhibition could contribute to the reversion of 5FU resistance (Fig. 2, D-F). Interestingly, similar results were obtained after treatment of the cells with 217505 (Fig. 2, G-I). Overall, these data suggest that blocking the P38/Hsp27/ CREB pathway can help reverse HCC cell resistance.

CD13 Promotes HCC Cell Resistance to 5FU by Activating P38/Hsp27/CREB Signaling. A previous study indicated that CD13 and the P38/Hsp27/CREB pathway are involved in the drug resistance of liver cancer cells. Thus, we considered the possibility that CD13 activates the P38/Hsp27/

A
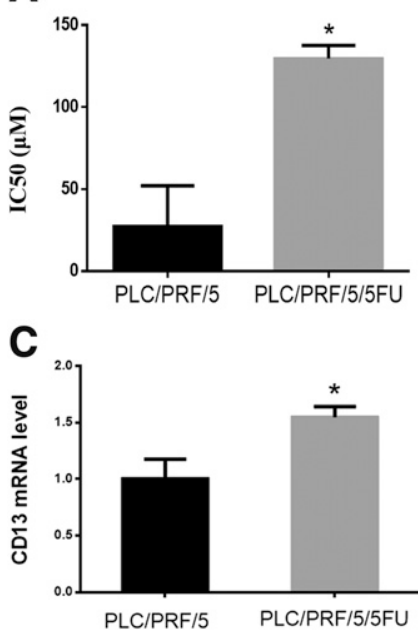

B

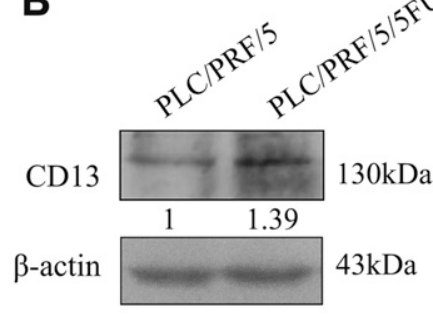

Fig. 1. Establishment of a resistant HCC cell line and determination of the expression level of CD13. (A) IC $\mathrm{IC}_{50}$ values were detected by MTT assay. Data are presented as means \pm S.D. $(n=3)$. (B) CD13 expression was determined by using Western blot. A representative immunoblot from three independent experiments giving similar results is shown, and $\beta$-actin is used as a normalizing protein. (C) CD13 mRNA levels were detected by qPCR. Data are represented as means \pm S.D. $(n=3)$. Statistical analysis was conducted by using a Student's $t$ test. ${ }^{*} P \leq 0.05$. 
D

A

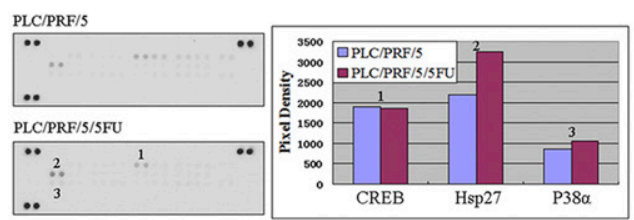

B

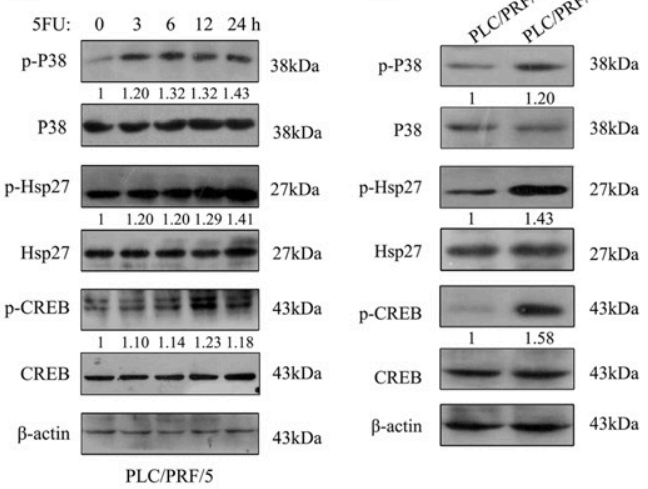

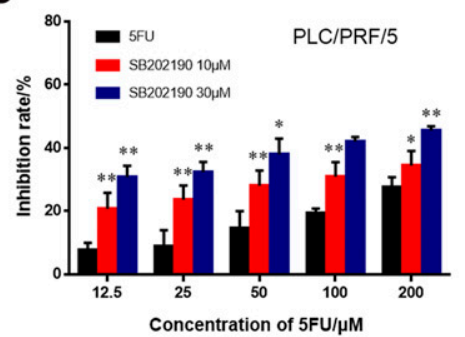

$\mathbf{F}$

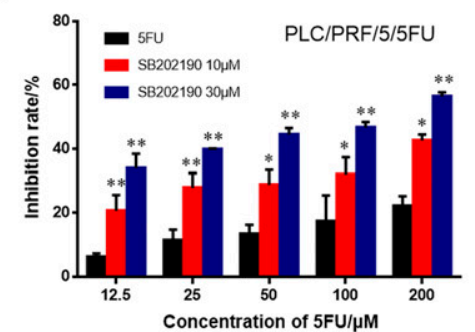

H

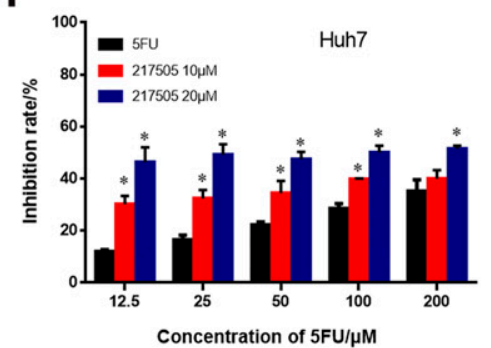

E

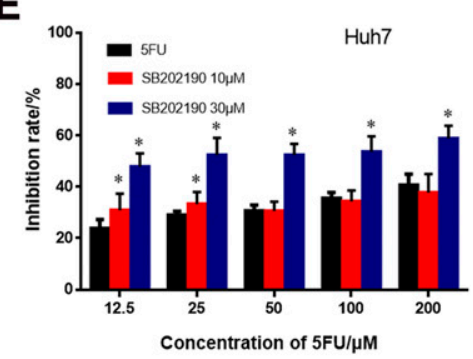

G

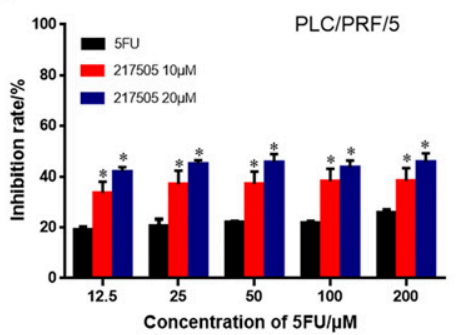

I

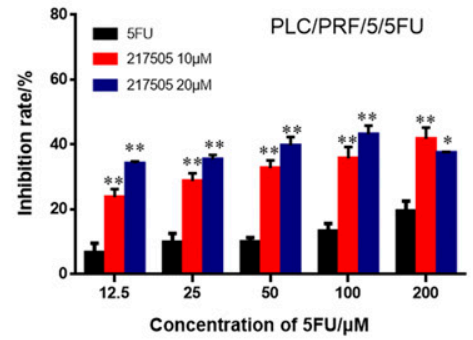

Fig. 2. P38/Hsp27/CREB pathway participates in HCC cell chemoresistance. (A) Phosphorylation of MAPK signaling pathway-related proteins were examined by protein ChIP assay in PLC/PRF/5 and PLC/PRF/5/5FU cells. (B) PLC/PRF/5 cells were treated with $5 \mathrm{FU}$. Immunoblot was performed to detect the phosphorylation of P38, Hsp27, and CREB. (C) The protein expression of p-P38, p-Hsp27, and p-CREB was determined by Western blot in $\mathrm{PLC} / \mathrm{PRF} / 5$ and PLC/PRF/5/5FU cells. A representative immunoblot from three independent experiments giving similar results is shown, and total protein was used as an internal control. The viability of PLC/PRF/5 (D), Huh7 (E), and PLC/PRF/5/5FU (F) cells treated with the P38 inhibitor SB202190 and 5FU for 48 hours was examined by MTT assay. PLC/PRF/5 (G), Huh7 (H), and PLC/PRF/5/5FU (I) cells were treated with the CREB inhibitor 217505 and 5FU for 48 hours, and cell viability was measured by MTT assay. Data are represented as means \pm S.D. $(n=3)$. Statistical analysis was conducted by one-way ANOVA followed by Dunnett's test (D-I). $* P \leq 0.05$; $* * \leq 0.01$.

CREB pathway to induce the drug resistance of HCC cells. To explore this possibility, we detected the effects of ubenimex on the P38/Hsp27/CREB pathway. As expected, ubenimex could downregulate p-P38, p-Hsp27, and p-CREB expression levels in HCC cells (Fig. 3, A and B). To confirm the relationship between CD13 and P38/Hsp27/CREB pathway, we constructed PLC/PRF/5 cells exhibiting stable CD13 overexpression or knockdown. As expected, the Western blot results showed that the phosphorylation levels of P38, Hsp27, and CREB are upregulated in PLC/PRF/5/CD13 cells compared with those in parental cells. In addition, knockdown of CD13 downregulated phosphorylation levels, which suggests that CD13 functions upstream of the P38/Hsp27/CREB pathway to facilitate HCC cell resistance (Fig. 3C). A recent study indicated that P38 could regulate the expression and phosphorylation level of Hsp27 (Guay et al., 1997; He et al., 2012). We examined the relationship between P38/Hsp27 and CREB by treating PLC/PRF/ 5 and Huh7 cells with SB202190. Western blot results suggested that the expression of p-Hsp27 and p-CREB is downregulated after SB202190 treatment in a dose-dependent manner, thereby indicating that CREB is located downstream of P38/Hsp27 (Fig. 3, D and E). These results reveal that the CD13-stimulated P38/Hsp27/ CREB cascade pathway accounts for HCC cell resistance.
CREB-Mediated Autophagy Facilitates HCC Cell Resistance to 5FU via Binding to the ATG7 Promoter Region. Differences in mRNA level between PLC/PRF/5 and PLC/PRF/5/CD13 cells were analyzed by RNA transcriptome ChIP technology (Supplemental Fig. 2, A-C) to explore the mechanism of HCC cell resistance further. We found that ATG7 mRNA expression is significantly higher in PLC/PRF/5/ CD13 cells than in parental cells. ATG7 mRNA and protein levels were determined in PLC/PRF/5 and PLC/PRF/5/CD13 cells by qPCR and Western blot, respectively. Data indicated that ATG7 is obviously upregulated in PLC/PRF/5/CD13 cells compared with that in parental cells (Fig. 4, A and B). CREB reportedly serves as a transcriptional factor that promotes gene transcription (Lamprecht, 1999). Given the role of CREB and its transcriptional activation of ATG7, we deduced that CREB may directly bind to the ATG7 promoter region. We designed a series of primers flanking the $A T G 7$ promoter from -2074 to +100 . As shown in Supplemental Fig. 3, CREB could bind to the -1809 to -1412 promoter region of ATG7. Moreover, a significant increase in the binding activity of CREB to the ATG7 promoter region was observed in PLC/ $\mathrm{PRF} / 5 / 5 \mathrm{FU}$ cells compared with that in parental cells (Fig. 4, C 
A

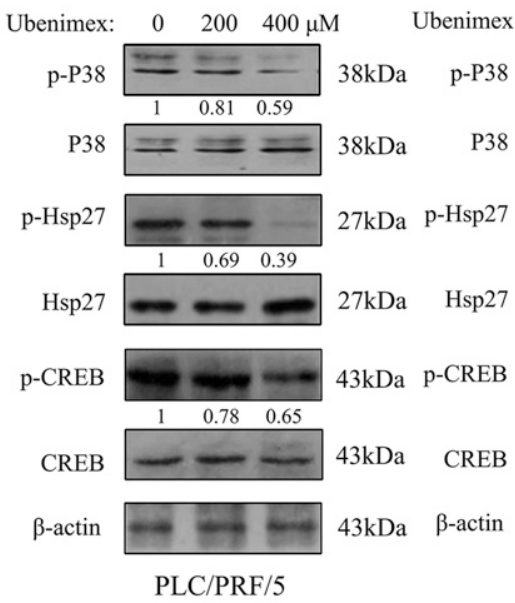

D

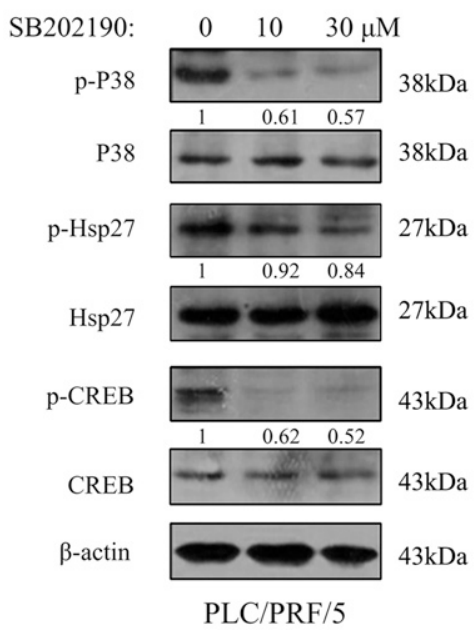

C

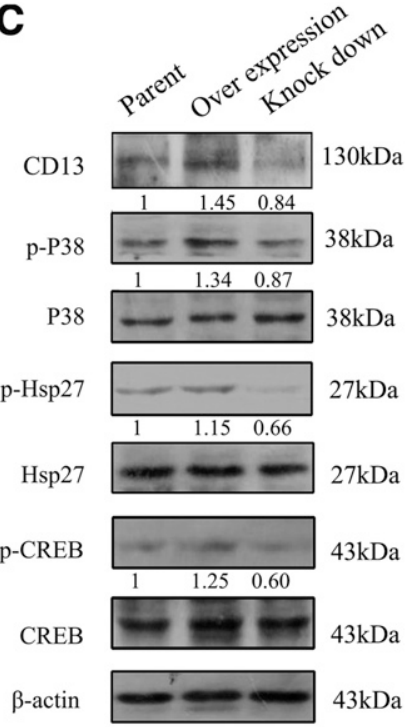

E

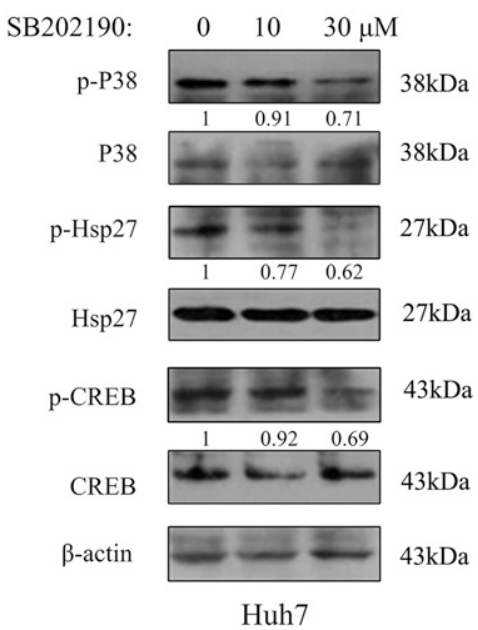

Fig. 3. P38/Hsp27/CREB pathway functions downstream of CD13. PLC/PRF/5 (A) and Huh7 (B) cells were treated with ubenimex for 30 hours, and the phosphorylation of signaling pathway-related proteins was detected by Western blot. (C) $\mathrm{PLC} / \mathrm{PRF} / 5$ cells were infected with lentivirus. After puromycin screening, PLC/ $\mathrm{PRF} / 5$ cells exhibiting stable CD13 overexpression or knockdown were obtained. The expression levels of p-P38, p-Hsp27, and $\mathrm{p}$-CREB were detected by treatment of PLC/PRF/5 (D) and Huh7 (E) cells with the P38 inhibitor SB202190 for 24 hours followed by Western blot to measure protein phosphorylation. A representative immunoblot from three independent experiments giving similar results is shown, and $\beta$-actin or total protein was used as a protein loading control. and D). Western blot results further showed that ATG7 expression in resistant cells is higher than that in parental cells.

ATG7 is an E-1 enzyme that activates ubiquitin-like proteins, such as ATG12 and ATG8, and promotes their transfer to an E-2 enzyme, which is crucial for autophagosome formation in the canonical pathway (Zhu et al., 2019). Thus, we hypothesized that CREB-mediated autophagy may be involved in HCC cell resistance. Protein expression associated with autophagy was detected by Western blot, and results showed that microtubule-associated protein light chain 3 II expression is downregulated, whereas sequestosome 1 expression is upregulated in resistant cells. This finding suggests that the autophagy process may facilitate HCC cell resistance (Fig. 4E). The CREB inhibitor 217505 was then employed to determine whether CREB is required for the expression of ATG7 and autophagy. The data indicated that ATG7 expression and autophagy are upregulated by $5 \mathrm{FU}$ treatment but neutralized by 217505 treatment in PLC/PRF/5 and Huh7 cells (Fig. 4, F and G). PLC/PRF/5 cells were transfected with the stubRFP-sensGFP-LC3B lentivirus to mark LC3B-II and explore whether CREB mediates autophagy. The infected cells were treated with $5 \mathrm{FU}$ or 217505 to detect the number of autophagosomes and autolysosomes formed. The results demonstrated that $5 \mathrm{FU}$ could induce the autophagy process and that this effect could be neutralized by 217505 . These results indicate that CREB participates in autophagy (Fig. 4H). We confirmed the relationship between autophagy and resistance by MTT assay. PLC/PRF/5 and PLC/PRF/5/ $5 \mathrm{FU}$ cells were treated with the autophagy inhibitor 3methyladenine (3-MA) and 5FU. The data indicated that 3MA could similarly sensitize resistant and parental cells to 5FU (Fig. 4I). These results indicate that CREB-mediated ATG7 expression and autophagy could promote HCC cell resistance to $5 \mathrm{FU}$.

Combination of Ubenimex and 5FU Inhibits Nude Mice Tumor Growth In Vivo. In this study, nude mice bearing tumor cells were used to confirm whether inhibition of CD13 enhances the antitumor effect of 5FU in vivo. Approximately $1 \times 10^{\wedge} 7 \mathrm{PLC} / \mathrm{PRF} / 5$ cells were administered into the right armpit of the nude mice. The results obtained showed that the combination of $5 \mathrm{FU}$ and ubenimex markedly inhibits 
A

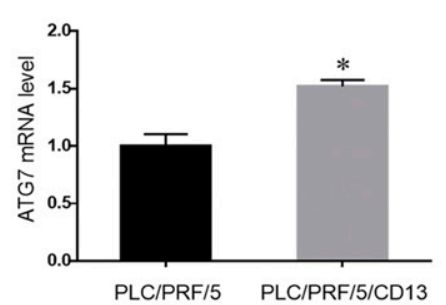

D

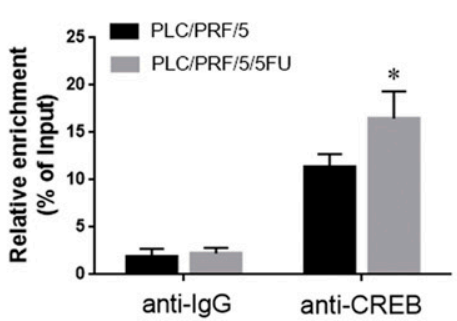

B

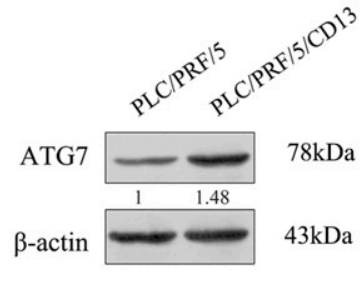

E

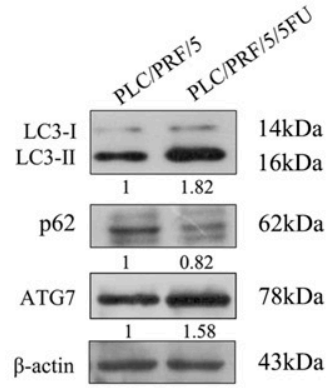

C

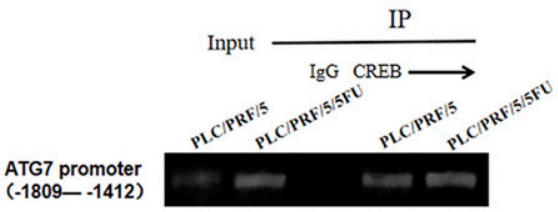

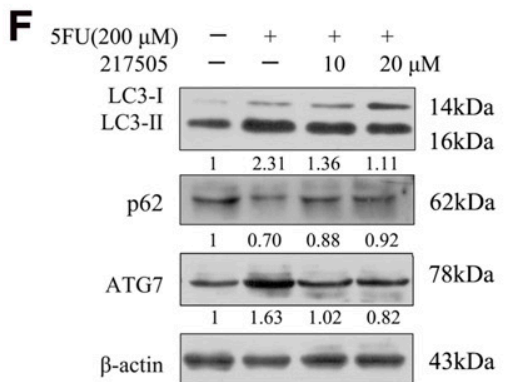

$\mathrm{PLC} / \mathrm{PRF} / 5$
G

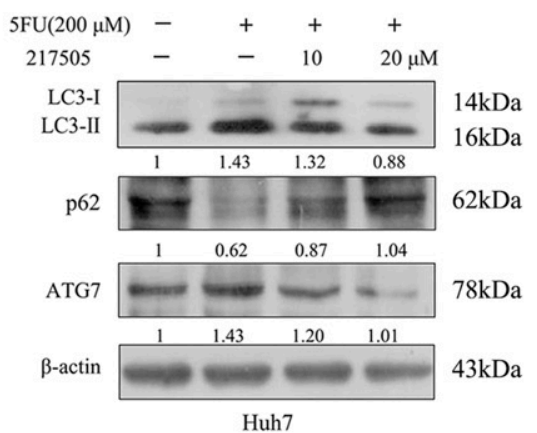

I

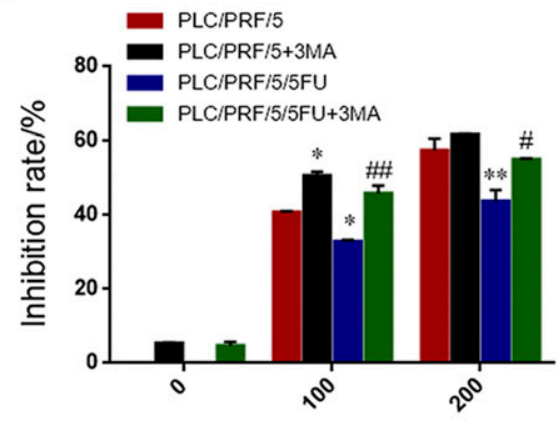

Concentration of $5 \mathrm{FU} / \mu \mathrm{M}$

H
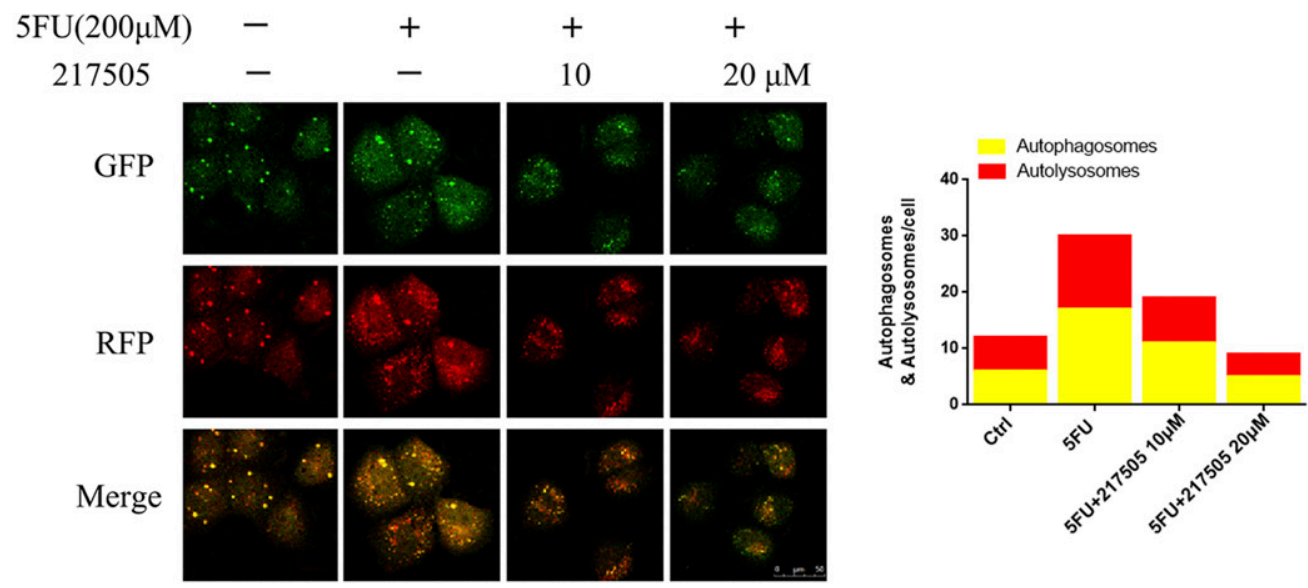

Fig. 4. Transcriptional factor CREB combines with the promoter of the autophagy-related protein ATG7. ATG7 mRNA (A) and protein (B) levels in PLC/ $\mathrm{PRF} / 5$ and PLC/PRF/5/CD13 cells were analyzed by qPCR or Western blot. ChIP assay covering the region of the ATG7 promoter from - 1809 to - 1412 was performed to measure the binding activity of CREB to the $A T G 7$ promoter in PLC/PRF/5 and PLC/PRF/5/5FU cells (C). IgG was used as a negative control. Enrichment of the ATG7 promoters was quantified by qRT-PCR, and the result is presented as a percentage relative to chromatin input (D). Data represent the means \pm S.D. of three independent experiments. $* P<0.05$. (E) Protein expression levels of ATG7, LC3-II, and p62 were detected by Western blot in PLC/PRF/5 and PLC/PRF/5/CD13 cells. PLC/PRF/5 (F) and Huh7 (G) cells were pretreated with the CREB inhibitor 217505 for 2 hours and then with $5 \mathrm{FU}$ treatment of 24 hours. Then autophagy-related protein expression was examined by Western blot. $\beta$-actin was used as a protein loading control. (H) PLC/PRF/5 cells were infected with the stubRFP-sensGFP-LC3B lentivirus, pretreated with 217505 for 2 hours, and then treated with $5 \mathrm{FU}$ for 24 hours. Quantification of autophagosome and autolysosome formation representing puncta staining sites per cell (Scale bar, $50 \mu \mathrm{m}$ ). Experiments were performed in triplicate. ${ }^{*} P P \leq 0.01$ vs. the control; ${ }^{\#} P \leq 0.05 ;{ }^{\# \#} P \leq 0.01$ vs. $5 \mathrm{FU}$. (I) PLC/PRF/5 and PLC/PRF/5/5FU cells were administered with 3-MA and 5FU for 48 hours and then were subjected to MTT assay. Data are represented as means \pm S.D. of three independent experiments. ${ }^{*} P \leq 0.05 ;{ }^{* *} P \leq 0.01$ vs. PLC/PRF/ $;{ }^{\#} P \leq 0.05 ;{ }^{\# \#} P \leq 0.01$ vs. PLC/PRF/5/5FU. Statistical analysis was carried out using a Student's $t$ test (A and D) and two-way ANOVA followed by Bonferroni's test (H-I). 
the growth of tumor tissue compared with $5 \mathrm{FU}$ or ubenimex alone (Fig. 5, A-D). We then detected protein expression in the tumor tissues. Western blot indicated that, whereas 5FU could induce the expression of CD13, p-CREB, ATG7, and autophagy, ubenimex could suppress this effect (Fig. 5E). Taken together, the data prove that CD13 overexpression could induce autophagy to promote $\mathrm{HCC}$ cell resistance to $5 \mathrm{FU}$ in vivo.

\section{Discussion}

HCC, which is characterized by a high degree of malignancy and poor prognosis, is the fifth most frequently occurring cancer worldwide (El-Serag and Mason, 1999; Mlynarsky et al., 2015). Although anticancer chemotherapeutic drugs for HCC have shown considerable efficacy in terms of prolonging life expectancy, chemoresistance is still often encountered. Chemoresistance reduces the effect of anticancer drugs and results in failure of $\mathrm{HCC}$ therapy (Fu et al., 2018). Thus, exploring the molecular mechanism underlying the development of HCC cell chemoresistance is an important endeavor. In this study, we found that CD13 inhibition could increase the sensitivity of HCC cells to 5FU and reverse their chemoresistance, thereby indicating that CD13 is a potential therapeutic target. Our results further showed that CD13 promotes the expression of ATG7 by activating the P38/ Hsp27/CREB signaling pathway to induce autophagy leading to $\mathrm{HCC}$ cell resistance.

CD13, a biomarker of human liver CSCs, is related to cancer multidrug resistance, recurrence, and metastasis (Haraguchi et al., 2010). In this study, the resistant cell line PLC/PRF/5/ $5 \mathrm{FU}$ was employed to detect the relationship between CD13 and resistance. Western blot results showed that the expression of CD13 is highly elevated in resistant cells compared with that in parental cells. Moreover, our previous study revealed that ubenimex and neutralizing antibody could enhance the sensitivity of tumor cells to cytotoxic agents. CD13 overexpression or knockdown affects the sensitivity of cells to cytotoxic agents (Zhang et al., 2018). The compound BC-02, which could decompose into 5FU and ubenimex, showed significant suppression of the self-renewal and malignant proliferation of liver CSCs compared with the control, ubenimex, $5 \mathrm{FU}$, and even $5 \mathrm{FU}+$ ubenimex treatments, thus suggesting that the combination of 5FU and a CD13 inhibitor could reverse tumor cell resistance (Dou et al., 2017). The CD13 inhibitor 4cc also synergizes the antitumor effects of $5 \mathrm{FU}$ in human liver cancer cells via ROS-mediated drug resistance inhibition and concurrent activation of the mitochondrial pathway during apoptosis (Sun et al., 2015). Overall, we deduced that CD13 may contribute to chemoresistance of HCC cells to $5 \mathrm{FU}$.

To verify the molecular mechanism through which CD13 participates in HCC cell resistance, we employed protein chip technology data to show that the phosphorylation levels of P38, Hsp27, and CREB are obviously upregulated in resistant cells compared with those in parental cells. P38 protein kinase is a member of the mitogen-activated protein kinase (MAPK) family (Rincón and Davis, 2009). P38 MAPK is an important signaling pathway involved in the growth, survival, differentiation, and inflammatory responses of cells and can be
A

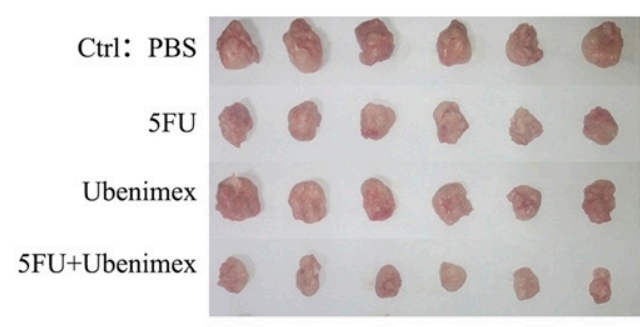

B

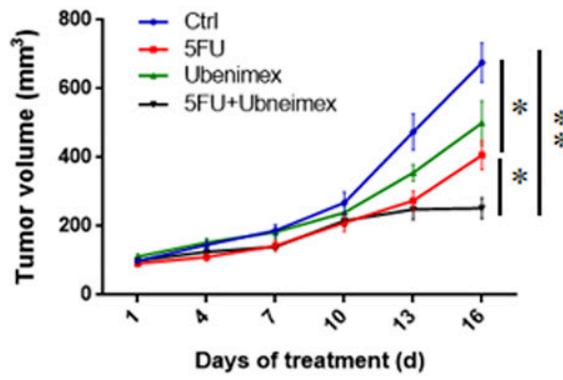

C

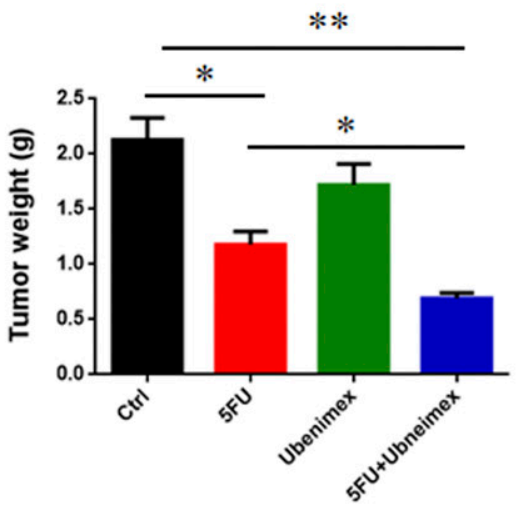

D

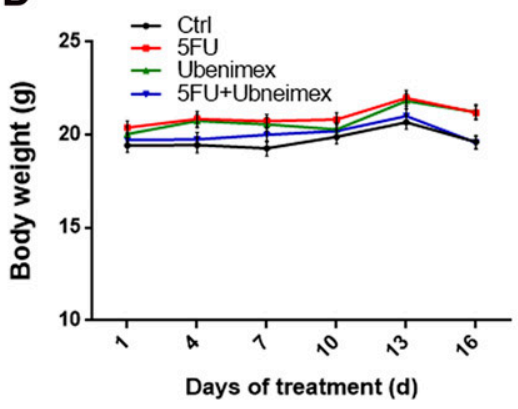

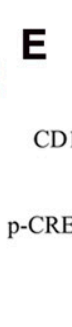

CREB

LC3-I LC3-II

ATG7

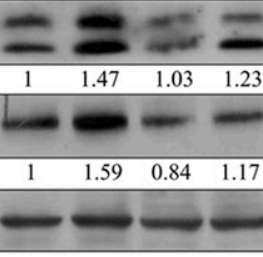

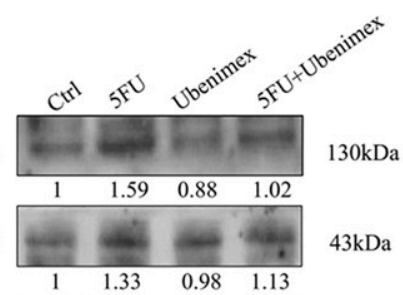

$43 \mathrm{kDa}$

$14 \mathrm{kDa}$ $16 \mathrm{kDa}$ $78 \mathrm{kDa}$ $43 \mathrm{kDa}$

Fig. 5. Combination of ubenimex and $5 \mathrm{FU}$ inhibits the growth of tumor cells in vivo. PLC/PRF/5 cells were injected into the right armpit of nude mice. After tumor formation, the mice were randomly divided into four groups $(n=6)$ : Ctrl, $5 \mathrm{FU}$ ( $25 \mathrm{mg} / \mathrm{kg}$ per day), ubenimex (74 mg/kg per day), and ubenimex $+5 \mathrm{FU}$. The body weights and tumor volumes of the mice were monitored (D). After 2 weeks, all mice were sacrificed and dissected, and tumor tissue were weighed (A-C). (E) Protein expression in tumor samples of the Ctrl, $5 \mathrm{FU}$, ubenimex, and ubenimex +5 FU groups was detected by Western blot. A representative immunoblot from three independent experiments giving similar results is shown, and $\beta$-actin or total protein was used as a protein loading control. Data are represented as means \pm S.D. $* P \leq 0.05$; ** $P \leq 0.01$ (B and C two-tailed $t$ test). Ctrl, control. 
activated by a variety of cellular stresses, including inflammatory cytokines, endotoxins, UV light, and growth factors. The existence of a new mechanism of resistance to camptothecin-related drugs has been reported in this mechanism; MAPK14/p38 $\alpha$ is activated upon SN38 induction and triggers survival promoting autophagy to protect tumor cells against the cytotoxic effects of drugs (Paillas et al., 2012; Hui et al., 2014; Yu et al., 2014; Zhou et al., 2019). Hsp27, a member of the small heat shock protein family, is induced by stress and protects against heat shock, hypertonic stress, oxidative stress, and other forms of cellular injury in numerous cell types (Xu et al., 2013). Overexpression of Hsp27 has been associated with poor prognosis for astrocytic brain tumor (Golembieski et al., 2008), breast cancer (Kang et al., 2008), ovarian carcinoma (Arts et al., 1999), and HCC (Yang et al., 2010). A previous study demonstrated that Hsp27 expression is a prognostic marker involved in the histologic grade and survival of patients with HCC (King et al., 2000; Feng et al., 2005). Yang et al., 2010 revealed that miR-17-5p promotes the migration of human HCC cells through the P38 mitogenactivated protein kinase-Hsp27 pathway, which suggests that Hsp27 may function downstream of P38 (Guay et al., 1997; Xu et al., 2013). Taken together, our findings suggest that P38/ Hsp27 is associated with HCC cell resistance.

CREB is a nuclear transcriptional factor that regulates target gene transcription activity associated with cell survival and death (Mayr and Montminy, 2001; Wang et al., 2013a,b). It is regulated by multiple protein kinases and protein phosphatases. Overexpression and hyperactivation of CREB1 are often observed in acute myeloid leukemia and several human solid malignancies, including breast, lung, ovary, and prostate carcinomas. Inhibition of CREB1 in several human cancer cell lines induces apoptosis and suppression of cell proliferation, indicating its critical role as a proto-oncogene (Wang et al., 2013b). In the current study, we found that CREB inhibition increases the sensitivity of HCC cells to 5FU. Thus, we speculate that CD13 may regulate the activity of CREB to induce HCC cell resistance through the P38/Hsp27 pathway.

The genes of peptide neurotransmitters, neurotrophic factors, and even transcriptional factors in specific brain regions are downstream targets of CREB (Pandey, 2003; Pandey et al., 2005; Zhang et al., 2005). However, other target genes of CREB have not been reported. Thus, we speculate that CD13 activates CREB to promote downstream protein expression through the P38/Hsp27 pathway and facilitate HCC cell resistance. Using the ChIP method, we found that CREB binds to the -1809 to -1412 region of the ATG7 promoter. The E1-like enzyme ATG7 coordinates with the E2-like enzyme ATG10 to mediate the conjugation of ATG12 to ATG5 and promote lipid phosphatidylethanolamine formation (Luo et al., 2016). ATG7 deletion in mouse liver facilitates HCC development (Takamura et al., 2011). By contrast, inhibition of ATG7 suppresses rat sarcoma (RAS)-mediated breast tumor growth (Guo et al., 2011), and high ATG7 expression indicates poor prognosis for breast cancer (Desai et al., 2013). Upregulation of ATG7 has been detected in many HCC samples and appears to facilitate tumor cell survival during metabolic stress (Luo et al., 2016). Our current study showed that CREB binds to the ATG7 promoter region to induce autophagy. We also demonstrated that autophagy inhibition could enhance the cytotoxicity of 5FU toward HCC cells.
Our in vivo nude mice xenograft model assay confirmed this conclusion.

ROS upregulation is involved in CD13 suppression-induced cell death. That work mainly focused on ROS. In the present study, we observed that CD13 inhibition can reverse HCC cell resistance by blocking CREB-mediated autophagy. However, because CREB is a transcriptional factor that may bind to other target genes influencing HCC cell resistance, further research on this topic is warranted.

\section{Authorship Contributions}

Participated in research design: Y. Zhao, Wu, Yan, X. Wang. Conducted experiments: Y. Zhao, Wu, Xing, Ma, Ji, Xu, Jiang Performed data analysis: X. Zhao, S. Wang, Fang, Zhang.

Wrote or contributed to the writing of the manuscript: Y. Zhao, $\mathrm{X}$. Wang.

\section{References}

Arts HJ, Hollema H, Lemstra W, Willemse PH, De Vries EG, Kampinga HH, and Van der Zee AG (1999) Heat-shock-protein-27 (hsp27) expression in ovarian carcinoma: relation in response to chemotherapy and prognosis. Int $J$ Cancer 84:234-238.

Chen R, Dai RY, Duan CY, Liu YP, Chen SK, Yan DM, Chen CN, Wei M, and Li H (2011) Unfolded protein response suppresses cisplatin-induced apoptosis via autophagy regulation in human hepatocellular carcinoma cells. Folia Biol (Praha) 57:87-95.

Desai S, Liu Z, Yao J, Patel N, Chen J, Wu Y, Ahn EEY, Fodstad O, and Tan M (2013) Heat shock factor 1 (HSF1) controls chemoresistance and autophagy through transcriptional regulation of autophagy-related protein 7 (ATG7). J Biol Chem 288: 9165-9176.

Dou C, Fang C, Zhao Y, Fu X, Zhang Y, Zhu D, Wu H, Liu H, Zhang J, Xu W, et al. (2017) BC-02 eradicates liver cancer stem cells by upregulating the ROSdependent DNA damage. Int $J$ Oncol 51:1775-1784.

El-Serag HB and Mason AC (1999) Rising incidence of hepatocellular carcinoma in the United States. N Engl J Med 340:745-750.

Feng JT, Liu YK, Song HY, Dai Z, Qin LX, Almofti MR, Fang CY, Lu HJ, Yang PY, and Tang ZY (2005) Heat-shock protein 27: a potential biomarker for hepatocellular carcinoma identified by serum proteome analysis. Proteomics 5:4581-4588.

Fu XT, Song K, Zhou J, Shi YH, Liu WR, Tian MX, Jin L, Shi GM, Gao Q, Ding ZB, et al. (2018) Autophagy activation contributes to glutathione transferase $\mathrm{Mu} 1$ mediated chemoresistance in hepatocellular carcinoma. Oncol Lett 16:346-352.

Golembieski WA, Thomas SL, Schultz CR, Yunker CK, McClung HM, Lemke N, Cazacu S, Barker T, Sage EH, Brodie C, et al. (2008) HSP27 mediates SPARCinduced changes in glioma morphology, migration, and invasion. Glia 56: 1061-1075.

Guay J, Lambert H, Gingras-Breton G, Lavoie JN, Huot J, and Landry J (1997) Regulation of actin filament dynamics by p38 map kinase-mediated phosphorylation of heat shock protein 27. J Cell Sci 110:357-368.

Guo JY, Chen HY, Mathew R, Fan J, Strohecker AM, Karsli-Uzunbas G, Kamphorst JJ, Chen G, Lemons JM, Karantza V, et al. (2011) Activated Ras requires autophagy to maintain oxidative metabolism and tumorigenesis. Genes Dev 25: 460-470.

Guo Q, Sui ZG, Xu W, Quan XH, Sun JL, Li X, Ji HY, and Jing FB (2017) Ubenimex suppresses Pim-3 kinase expression by targeting CD13 to reverse MDR in HCC cells. Oncotarget 8:72652-72665.

Haraguchi N, Ishii H, Mimori K, Tanaka F, Ohkuma M, Kim HM, Akita H, Takiuch D, Hatano H, Nagano H, et al. (2010) CD13 is a therapeutic target in human liver cancer stem cells. J Clin Invest 120:3326-3339.

Hashida H, Takabayashi A, Kanai M, Adachi M, Kondo K, Kohno N, Yamaoka Y, and Miyake M (2002) Aminopeptidase $\mathrm{N}$ is involved in cell motility and angiogenesis: its clinical significance in human colon cancer. Gastroenterology 122: $376-386$

He J, Liu Z, Zheng Y, Qian J, Li H, Lu Y, Xu J, Hong B, Zhang M, Lin P, et al. (2012) p38 MAPK in myeloma cells regulates osteoclast and osteoblast activity and induces bone destruction. Cancer Res 72:6393-6402.

Hu Y, Zhang HR, Dong L, Xu MR, Zhang L, Ding WP, Zhang JQ, Lin J, Zhang YJ, Qiu BS, et al. (2019) Enhancing tumor chemotherapy and overcoming drug resistance through autophagy-mediated intracellular dissolution of zinc oxide nanoparticles. Nanoscale 11:11789-11807.

Huang F, Wang BR, and Wang YG (2018) Role of autophagy in tumorigenesis, metastasis, targeted therapy and drug resistance of hepatocellular carcinoma. World $J$ Gastroenterol 24:4643-4651.

Hui K, Yang Y, Shi K, Luo H, Duan J, An J, Wu P, Ci Y, Shi L, and Xu C (2014) The p38 MAPK-regulated PKD1/CREB/Bcl-2 pathway contributes to selenite-induced colorectal cancer cell apoptosis in vitro and in vivo. Cancer Lett 354:189-199.

Ishii K, Usui S, Sugimura Y, Yoshida S, Hioki T, Tatematsu M, Yamamoto H, and Hirano K (2001) Aminopeptidase N regulated by zinc in human prostate participates in tumor cell invasion. Int J Cancer 92:49-54.

Kang SH, Kang KW, Kim KH, Kwon B, Kim SK, Lee HY, Kong SY, Lee ES, Jang SG, and Yoo BC (2008) Upregulated HSP27 in human breast cancer cells reduces Herceptin susceptibility by increasing Her2 protein stability. BMC Cancer 8:286. King KL, Li AF, Chau GY, Chi CW, Wu CW, Huang CL, and Lui WY (2000) Prognostic significance of heat shock protein-27 expression in hepatocellular carcinoma and its relation to histologic grading and survival. Cancer 88:2464-2470.

Lamprecht R (1999) CREB: a message to remember. Cell Mol Life Sci 55:554-563. 
Levy JMM, Towers CG, and Thorburn A (2017) Targeting autophagy in cancer. Nat Rev Cancer 17:528-542.

Luo T, Fu J, Xu A, Su B, Ren Y, Li N, Zhu J, Zhao X, Dai R, Cao J, et al. (2016) PSMD10/gankyrin induces autophagy to promote tumor progression through cytoplasmic interaction with ATG7 and nuclear transactivation of ATG7 expression. Autophagy 12:1355-1371.

Mayr B and Montminy M (2001) Transcriptional regulation by the phosphorylationdependent factor CREB. Nat Rev Mol Cell Biol 2:599-609.

Mina-Osorio P (2008) The moonlighting enzyme CD13: old and new functions to target. Trends Mol Med 14:361-371.

Mlynarsky L, Menachem Y, and Shibolet O (2015) Treatment of hepatocellular carcinoma: steps forward but still a long way to go. World J Hepatol 7:566-574.

Nagano H, Ishii H, Marubashi S, Haraguchi N, Eguchi H, Doki Y, and Mori M (2012) Novel therapeutic target for cancer stem cells in hepatocellular carcinoma. $J$ Hepatobiliary Pancreat Sci 19:600-605.

O'Donovan TR, O'Sullivan GC, and McKenna SL (2011) Induction of autophagy by drug-resistant esophageal cancer cells promotes their survival and recovery following treatment with chemotherapeutics. Autophagy 7:509-524.

Paillas S, Causse A, Marzi L, de Medina P, Poirot M, Denis V, Vezzio-Vie N, Espert L, Arzouk H, Coquelle A, et al. (2012) MAPK14/p38 $\alpha$ confers irinotecan resistance to TP53-defective cells by inducing survival autophagy. Autophagy 8:1098-1112.

Pan H, Wang Z, Jiang L, Sui X, You L, Shou J, Jing Z, Xie J, Ge W, Cai X, et al. (2014) Autophagy inhibition sensitizes hepatocellular carcinoma to the multikinase inhibitor linifanib. Sci Rep 4:6683.

Pandey SC (2003) Anxiety and alcohol abuse disorders: a common role for CREB and its target, the neuropeptide Y gene. Trends Pharmacol Sci 24:456-460.

Pandey SC, Chartoff EH, Carlezon WA Jr., Zou J, Zhang H, Kreibich AS, Blendy JA, and Crews FT (2005) CREB gene transcription factors: role in molecular mechanisms of alcohol and drug addiction. Alcohol Clin Exp Res 29:176-184.

Raza A and Sood GK (2014) Hepatocellular carcinoma review: current treatment, and evidence-based medicine. World J Gastroenterol 20:4115-4127.

Rincón M and Davis RJ (2009) Regulation of the immune response by stressactivated protein kinases. Immunol Rev 228:212-224.

Sato Y (2003) Aminopeptidases and angiogenesis. Endothelium 10:287-290.

Sun ZP, Zhang J, Shi LH, Zhang XR, Duan Y, Xu WF, Dai G, and Wang XJ (2015) Aminopeptidase $\mathrm{N}$ inhibitor 4cc synergizes antitumor effects of 5-fluorouracil on human liver cancer cells through ROS-dependent CD13 inhibition. Biomed Pharmacother 76:65-72.

Takamura A, Komatsu M, Hara T, Sakamoto A, Kishi C, Waguri S, Eishi Y, Hino O, Tanaka K, and Mizushima N (2011) Autophagy-deficient mice develop multiple liver tumors. Genes Dev 25:795-800.

Wang P, Huang S, Wang F, Ren Y, Hehir M, Wang X, and Cai J (2013a) Cyclic AMPresponse element regulated cell cycle arrests in cancer cells. PLoS One 8:e65661.

Wang Y, Hu Z, Liu Z, Chen R, Peng H, Guo J, Chen X, and Zhang H (2013b) MTOR inhibition attenuates DNA damage and apoptosis through autophagy-mediated suppression of CREB1. Autophagy 9:2069-2086.
Wei KR, Yu X, Zheng RS, Peng XB, Zhang SW, Ji MF, Liang ZH, Ou ZX, and Chen WQ (2014) Incidence and mortality of liver cancer in China, 2010. Chin J Cancer 33:388-394.

Xu N, Zhang J, Shen C, Luo Y, Xia L, Xue F, and Xia Q (2012) Cisplatin-induced downregulation of miR-199a-5p increases drug resistance by activating autophagy in HCC cell. Biochem Biophys Res Commun 423:826-831.

Xu Y, Diao Y, Qi S, Pan X, Wang Q, Xin Y, Cao X, Ruan J, Zhao Z, Luo L, et al. (2013) Phosphorylated Hsp27 activates ATM-dependent p53 signaling and mediates the resistance of MCF-7 cells to doxorubicin-induced apoptosis. Cell Signal 25: 1176-1185.

Yamashita M, Wada H, Eguchi $H$, Ogawa H, Yamada D, Noda T, Asaoka T, Kawamoto K, Gotoh K, Umeshita K, et al. (2016) A CD13 inhibitor, ubenimex, synergistically enhances the effects of anticancer drugs in hepatocellular carcinoma. Int J Oncol 49:89-98.

Yang F, Yin Y, Wang F, Wang Y, Zhang L, Tang Y, and Sun S (2010) miR-17-5p promotes migration of human hepatocellular carcinoma cells through the p38 mitogen-activated protein kinase-heat shock protein 27 pathway. Hepatology 51:1614-1623.

Yu L, Yuan X, Wang D, Barakat B, Williams ED, and Hannigan GE (2014) Selective regulation of p38 $\beta$ protein and signaling by integrin-linked kinase mediates bladder cancer cell migration. Oncogene 33:690-701.

Zhang J, Fang C, Qu M, Wu H, Wang X, Zhang H, Ma H, Zhang Z, Huang Y, Shi L, et al. (2018) CD13 inhibition enhances cytotoxic effect of chemotherapy agents. Front Pharmacol 9:1042.

Zhang W, Zhao G, Wei K, Zhang Q, Ma W, Wu Q, Zhang T, Kong D, Li Q, and Song T (2015) Adjuvant sorafenib therapy in patients with resected hepatocellular carcinoma: evaluation of predictive factors. Med Oncol 32:107.

Zhang X, Odom DT, Koo SH, Conkright MD, Canettieri G, Best J, Chen H, Jenner R, Herbolsheimer E, Jacobsen E, et al. (2005) Genome-wide analysis of cAMPresponse element binding protein occupancy, phosphorylation, and target gene activation in human tissues. Proc Natl Acad Sci USA 102:4459-4464.

Zhou S, Li Y, Lu J, Chen C, Wang W, Wang L, Zhang Z, Dong Z, and Tang F (2019) Nuclear factor-erythroid 2-related factor 3 (NRF3) is low expressed in colorectal cancer and its down-regulation promotes colorectal cancer malignance through activating EGFR and p38/MAPK. Am J Cancer Res 9:511-528.

Zhu J, Tian Z, Li Y, Hua X, Zhang D, Li J, Jin H, Xu J, Chen W, Niu B, et al. (2019) ATG7 promotes bladder cancer invasion via autophagy-mediated increased ARHGDIB mRNA stability. Adv Sci (Weinh) 6:1801927.

Address correspondence to: Xuejian Wang, School of Pharmacy, Weifang Medical University, 7166 Baotong West Rd., Weifang, Shandong 261053, China. E-mail: wxj2901@126.com; or Fang Yan, School of Pharmacy, Weifang Medical University, 7166 Baotong West Rd., Weifang, Shandong 261053, China. E-mail: yanfang303@wfmc.edu.cn 